\title{
A novel NKX2-5 mutation in familial ventricular septal defect
}

\author{
JUAN WANG ${ }^{*}$, YUAN-FENG XIN ${ }^{2 *}$, XING-YUAN LIU ${ }^{3}$, \\ ZHONG-MIN LIU ${ }^{2}$, XIAO-ZHONG WANG ${ }^{4}$ and YI-QING YANG ${ }^{5}$
}

\begin{abstract}
Departments of ${ }^{1}$ Cardiology and ${ }^{2}$ Cardiothoracic Surgery, East Hospital, Tongji University School of Medicine, Shanghai 200120, ${ }^{3}$ Department of Pediatrics, Tongji Hospital, Tongji University School of Medicine, Shanghai 200065; Departments of ${ }^{4}$ Cardiac Surgery and ${ }^{5}$ Cardiovascular Research, Shanghai Chest Hospital, Medical College of Shanghai Jiaotong University, Shanghai 200030, P.R. China
\end{abstract}

Received October 5, 2010; Accepted November 29, 2010

DOI: $10.3892 /$ ijmm.2010.585

\begin{abstract}
Ventricular septal defect (VSD) is the most common cardiovascular malformation and an important contributor to the substantial morbidity and mortality in infancy. Growing evidence suggests that genetic defects play important roles in the pathogenesis of congenital VSD. However, VSD is of great genetic heterogeneity and the genetic basis for VSD in the majority of the patients remains largely unhnown. In this study, the entire coding region of the NKX2-5 gene, which encodes a homeodomain-containing transcription factor crucial to cardiogenesis, was initially sequenced in 136 unrelated patients with VSD. The relatives of a proband harboring the identified mutation and 200 unrelated control individuals were genotyped. The functional characteristic of the mutant transcription factor was analyzed in contrast to its wild-type counterpart using a luciferase reporter assay system. A novel heterozygous NKX2-5 mutation, p.P59A, was identified in a family with autosomal dominant inherited VSD. Absent in the 200 control individuals, the mutation was highly conserved evolutionarily and co-segregated with VSD in the family with complete penetrance. Functional analysis revealed that the p.P59A mutation of NKX2-5 was associated with a decreased transcriptional activity. These findings expand the spectrum of the mutations in NKX2-5 linked to VSD and provide new insight into the molecular mechanisms involved in VSD. The resuls of the present study may have potential implications in the genetic diagnosis and gene-specific therapy of this common childhood disease.
\end{abstract}

Correspondence to: Dr Yi-Qing Yang, Department of Cardiovascular Research, Shanghai Chest Hospital, Medical College of Shanghai Jiaotong University, 241 West Huaihai Road, Shanghai 200030, P.R. China

E-mail: yang99yang66@hotmail.com

${ }^{*}$ Contributed equally

Key words: ventricular septal defect, transcription factor, genetics

\section{Introduction}

Congenital heart disease is the most prevalent form of birth defect. It involves changes in the structure of the heart and in major blood vessels of a neonate, affects nearly $1 \%$ of newborns, and is the most common non-infectious cause of infant death resulting from birth abnormality. More than $29 \%$ of infants who die of a birth defect have a cardiovascular deformity (1). Congential heart disease, an abnormal development of the heart with dysmorphic features, is comprised of at least 18 distinct types with many additional anatomical variations, of which the ventricular septal defect (VSD) is the most common type. VSD occurs in approximately $50 \%$ of all children with congenital cardiovascular malformations, and accounts for 14 to $16 \%$ of the defects that require an invasive procedure within the first year of life $(1,2)$. Congenital VSD can occur alone or with other cardiac anomalies, such as atrial septal defect (ASD), Down syndrome, or tetralogy of Fallot. Regardless of other deformations that may accompany VSD, larger VSD may result in cardiac enlargement, congestive heart failure, pulmonary hypertension, Eisenmenger's syndrome, delayed fetal brain development, arrhythmias, and even sudden cardiac death in the absence of surgical or catheter based repair (3-7). Hence VSD is a leading cause of the markedly increased morbidity and mortality in infancy. Nevertheless, the molecular etiology in most VSD patients remains largely unknown $(8,9)$.

Developmentally, VSD is implicated in a heterogeneous, complex biological process associated with environmental and genetic risk factors (8-10). Accumulating evidence underlines the pivotal role of the homeobox-containing transcription factor NKX2-5, in cardiogenesis (11-16). The human NKX2-5 gene maps to chromosome $5 \mathrm{q} 34$ and consists of two exons encoding a protein of 324 amino acids. It is expressed during early cardiac morphogenesis and is indispensible for normal cardiac development (17). Therefore, NKX2-5 has been one of the preferred candidate genes in identifying the genetic determinants for structural congenital heart defects. Presently, more than 40 mutations of the $N K X 2-5$ gene have been identified in patients with a variety of congenital heart aberrations including VSD, ASD, conotruncal abnormalities such as tetralogy of Fallot, double outlet right ventricle, L-transposition of the 
great arteries, and hypoplastic left heart syndrome (18). These observations strongly suggest that NKX2-5 is important in the later stages of heart development and maturation in addition to its role in cardiac progenitor commitment and patterning in the developing heart (15).

In this study, the coding exons and exon/intron boundaries of NKX2-5 were initially sequenced in a cohort of 136 unrelated patients with congenital VSD and a novel heterozygous NKX2-5 mutation, p.P59A, was identified in a VSD patient with positive family history. Subsequently, genetic analysis of the family members demonstrated that the mutation co-segregated with an autosomal dominantly inherited form of VSD. Functional analysis revealed that the p.P59A mutation of NKX2-5 was associated with a decreased transcriptional activity. These findings expand the spectrum of mutations in NKX2-5 linked to VSD and provide new insight into the molecular mechanism involved in the pathogenesis of VSD.

\section{Materials and methods}

Study participants. A cohort of 136 unrelated patients with VSD was selected from the Chinese population. Subjects were evaluated by individual and familial history, review of the medical records, complete physical examination, a 12-lead electrocardiogram (ECG) and two-dimensional transthoracic echocardiography with color-flow Doppler. All patients had a classic form of VSD, with a defect diameter of $>3 \mathrm{~mm}$ and nearly all patients underwent cardiac catheterization and, if required, cardiac surgery. A total of 200 ethnically matched unrelated healthy individuals, which were derived from the general population, were used as controls to screen for the identified mutation in NKX2-5. Peripheral venous blood specimens were obtained from subjects and control individuals. The study protocol was reviewed and approved by the local institutional ethics committee and written informed consent was obtained from all participants or their guardians prior to investigation.

Genetic studies. Genomic DNA from all participants was extracted from blood lymphocytes with the Wizard Genomic DNA Purification Kit (Promega). Initially, the candidate gene NKX2-5 was screened in 136 unrelated patients with VSD and subsequently, genotyping of $N K X 2-5$ in the available relatives of the index patient carrying an identified mutation and the 200 ethnically matched unrelated healthy control individuals was conducted. The genomic reference DNA sequence of NKX2-5 was obtained from GenBank (accession No. NT_023133). The primer pairs used to amplify the complete coding region of $N K X 2-5$ by polymerase chain reaction (PCR) were designed with the on-line Primer 3 software (http:// frodo.wi.mit.edu) as follows: primer 1, forward 5'-CACGA TGCAGGGAAGCTG-3', and reverse 5'-AGTTTCTTGGGG ACGAAAGC-3' (the PCR product was 477 base pairs in size); primer 2, forward 5'-CCTCCACGAGGATCCCTTAC-3', and reverse 5'-CGAGTCCCCTAGGCATGG-3' (the product was 463 base pairs); primer 3, forward 5'-AGAACCGGCGCTACA AGTG-3', and reverse 5'-GAGTCAGGGAGCTGTTGAGG-3' (the product was 473 base pairs). Polymerase chain reaction (PCR) was carried out using HotStarTaq DNA polymerase (Qiagen) on a PE 9700 Thermal Cycler (Applied Biosystems), with standard conditions and concentrations of reagents.
Amplified products were analyzed on $1 \%$ agarose gels stained with ethidium bromide and purified with the QIAquick gel extraction kit (Qiagen). Both strands of each PCR product were sequenced with the BigDye ${ }^{\circledR}$ Terminator v3.1 Cycle Sequencing kit (Applied Biosystems) with an ABI PRISM 3130XL DNA Analyzer (Applied Biosystems). The sequencing primers used were previously designed for specific region sequencing. The DNA sequences were viewed and analyzed with the DNA Sequencing Analysis Software v5.1 (Applied Biosystems). The variant was validated by re-sequencing an independent PCR-generated amplicon from the subject and met our quality control thresholds with a call rate $>99 \%$.

Multiple sequence alignments. The multiple NKX2-5 protein sequences across mammals were aligned using the program MUSCLE (version 3.6).

Plasmids and site-directed mutagenesis. The recombinant expression plasmids pEFSA-NKX2-5 and atrial natriuretic peptide-luciferase reporter gene, which contains the 2600-bp 5'-flanking region of the atrial natriuretic peptide gene, namely ANP(-2600)-Luc, were kindly provided by Ichiro Shiojima, Chiba University School of Medicine. The identified mutation was introduced into the wild-type NKX2-5 using a QuickChange II XL site-directed mutagenesis kit (Stratagene) with a complementary pair of primers. The mutant was sequenced to confirm the desired mutation and to exclude any other sequence variations.

Reporter gene assays. COS-7 cells were cultured in Dulbecco's modified Eagle's medium supplemented with $10 \%$ fetal calf serum. The ANP(-2600)-Luc reporter construct and an internal control reporter plasmid pGL4.75 (hRluc/CMV, Promega) were used in transient transfection assays to examine the transcriptional activation function of the NKX2-5 mutant. COS-7 cells were transfected with $0.2 \mu \mathrm{g}$ of wild-type or mutant pEFSANKX2-5 expression vector, $0.2 \mu \mathrm{g}$ of ANP(-2600)-Luc reporter construct, and $0.04 \mu \mathrm{g}$ of pGL4.75 control reporter vector using PolyFect transfection reagent (Qiagen). For co-transfection experiments, $0.1 \mu \mathrm{g}$ of wild-type pEFSA-NKX2-5, $0.1 \mu \mathrm{g}$ of mutant pEFSA-NKX2-5, $0.2 \mu \mathrm{g}$ of ANP(-2600)-Luc, and $0.04 \mu \mathrm{g}$ of pGL4.75 were used. Firefly luciferase and Renilla luciferase activities were measured with the Dual-Glo luciferase assay system (Promega) $48 \mathrm{~h}$ after transfection. Three independent experiments were performed at minimum for wild-type and mutant $N K X 2-5$.

Statistics. Data are given as the mean \pm SD. Differences between the two groups were compared with the Student's $\mathrm{t}$-test for continuous variables and a 2 -tailed P-value $<0.05$ was considered to be statistically significant.

\section{Results}

Characteristics of the study subjects. A cohort of 136 unrelated patients with VSD was recruited and clinically evaluated against a cohort of 200 ethnically matched unrelated healthy individuals used as controls. None of them had overt traditional risk factors for VSD. The baseline clinical characteristics of the 136 unrelated patients with VSD are shown in Table I. 
Table I. Clinical characteristics of 136 unrelated patients with ventricular septal defect.

Number or mean value

Percentage or range

\section{Male:female}

$51: 85$

2.16

5.40

Age at the present study (years)

12

Positive family history

6

102

13

15

Muscular

Prevalence of VSDs with other defects

Isolated VSD

112

18

VSD and ASD

VSD, ASD and PDA

VSD, ASD and DORV

VSD and PDA

VSD and PS

Incidence of arrhythmias

Atrioventricular block

Atrial fibrillation

Treatment

Surgical repair

Percutaneous closure

Follow-up
60:100

0.17-12

0.17-16

8.82

4.41

75.00

9.56

11.03

82.35

13.24

1.47

1.47

0.74

0.74

5.15

2.21

84.56

2.94

12.50

VSD, ventricular septal defect; ASD, atrial septal defect; PDA, patent ductus arteriosus; DORV, double outlet right ventricle; PS, pulmonary stenosis.

NKX2-5 mutations. Direct sequencing of the coding regions of the $N K X 2-5$ gene was performed after PCR amplification of genomic DNA from the 136 unrelated VSD patients. A novel heterozygous mutation in $N K X 2-5$ was identified in 1 out of 136 patients. The total population prevalence of $N K X 2-5$ mutations based on the cohort patients was approximately $0.74 \%$. A substitution of guanine $(\mathrm{G})$ for cytosine $(\mathrm{C})$ in the first nucleotide of codon 59 of the NKX2-5 gene (c.175C $>\mathrm{G}$ ), predicting the transition of proline to alanine at amino acid position 59 (p.P59A), was identified in the proband from family 1 . The sequence chromatogram showing the detected heterozygous $N K X 2-5$ variation of $\mathrm{c} .175 \mathrm{C}>\mathrm{G}$ in comparison to control sequence was shown in Fig. 1. The variant was not present in 200 unrelated control individuals. Genetic scan of the family members displayed that the gene variant was present in all affected family members alive, but absent in the unaffected family members examined. Analysis of the pedigree demonstrated that the mutation co-segregated with VSD transmitted as an autosomal dominant trait in the family with complete penetrance. The pedigree structure of the family was illustrated in Fig. 2. The phenotypic characteristics and results of genetic screening of the affected pedigree members is listed in Table II. All 3 living patients underwent surgical repair of the VSDs.
Multiple cross-species alignments of the NKX2-5 protein sequences. A cross-species alignment of the NKX2-5 protein sequences showed that the altered amino acid P59 was highly

A

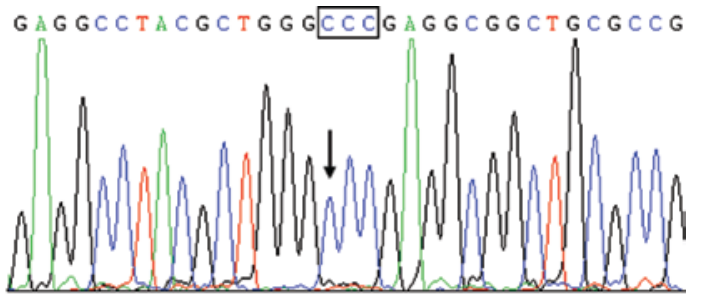

B

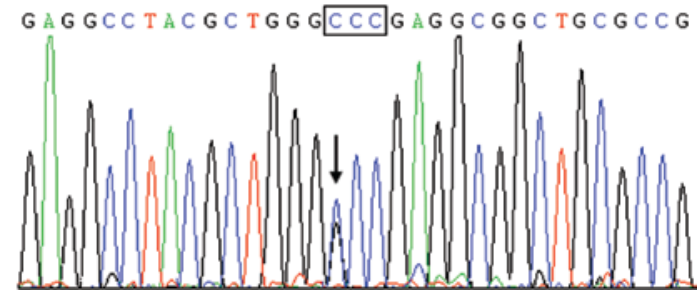

Figure 1. Sequence chromatogram showing the NKX2-5 mutation identified in family 1 . The arrow indicates the heterozygous nucleotides of $\mathrm{C} / \mathrm{G}$ in the proband from family 1 (B) or the homozygous nucleotides of $\mathrm{C} / \mathrm{C}$ in a control individual (A). The square denotes the nucleotides comprising a codon of NKX2-5. 
Table II. Phenotypic characteristics and status of the NKX2-5 mutation in the affected pedigree members.

\begin{tabular}{|c|c|c|c|c|c|c|c|}
\hline \multicolumn{4}{|c|}{ Subject Information } & \multicolumn{3}{|c|}{ Phenotype } & \multirow{2}{*}{$\frac{\text { Genotype }}{\text { P59A }}$} \\
\hline Identity & Gender & $\begin{array}{l}\text { Age at time of } \\
\text { study (years) }\end{array}$ & $\begin{array}{c}\text { Age at diagnosis of } \\
\text { VSD (years) }\end{array}$ & $\begin{array}{l}\text { VSD } \\
(\mathrm{mm})\end{array}$ & $\begin{array}{c}\text { Other structural } \\
\text { defects }\end{array}$ & AVB & \\
\hline II-2 & $\mathrm{F}$ & $58^{a}$ & 56 & 14 & ASD & + & N/A \\
\hline II-3 & M & $53^{\mathrm{a}}$ & 51 & 22 & PAS & + & N/A \\
\hline III-3 & $\mathrm{M}$ & 32 & 12 & 19 & - & - & $+/-$ \\
\hline IV-3 & M & 6 & 6 & 5 & - & - & $+/-$ \\
\hline IV-4 & $\mathrm{F}$ & 4 & 4 & 8 & - & - & $+/-$ \\
\hline
\end{tabular}

F, female; M, male; VSD, ventricular septal defect; N/A, not available; AVB, atrioventricular block; ASD, atrial septal defect; PAS, pulmonary artery stenosis. + indicates present and - denotes absent. ${ }^{\mathrm{a}} \mathrm{Age}$ at death.

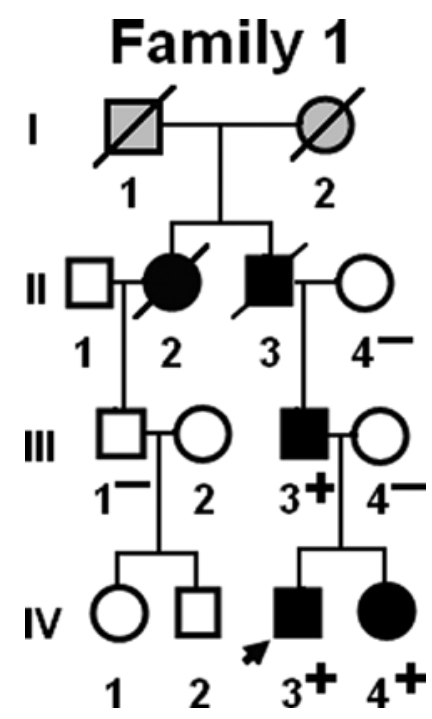

Figure 2. Pedigree structure of the family with the ventricular septal defect. Family members are identified by generations and numbers. Squares indicate male family members; circles, female members; symbols with a slash, the deceased members; closed symbols, affected members; open symbols, unaffected members; stippled symbols, members with phenotype undetermined; arrow, proband; +, carriers of the heterozygous mutations;--, non-carriers.

conserved evolutionarily among mammals as shown in Fig. 3, suggesting that the amino acid is functionally important.

Transcriptional activity of the NKX2-5 mutant. The transcriptional activation function of NKX2-5 in COS-7 cells was examined using one of its direct cardiac downstream target genes, $A N P$, as a luciferase reporter, and the activity of the $A N P$ promoter was presented as fold-activation of the firefly luciferase relative to Renilla luciferase. The same amounts of wild-type $(0.2 \mu \mathrm{g})$ and mutant $N K X 2-5(0.2 \mu \mathrm{g})$ activated the $A N P$ promoter by $\sim 6.6-$ and $\sim 3.2$-fold, respectively. When the same amount of wild-type $N K X 2-5(0.1 \mu \mathrm{g})$ was co-transfected with mutant $N K X 2-5(0.1 \mu \mathrm{g})$, the induced activation of the $A N P$ promoter was $\sim 4.5$-fold. These results suggest that mutant NKX2-5 has a significantly reduced activation activity compared with wild-type NKX2-5 (Fig. 4).

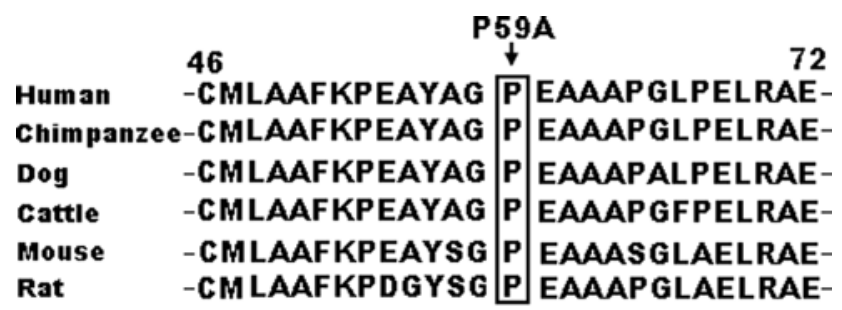

Figure 3. Alignment of multiple NKX2-5 protein sequences across species. The altered amino acid of P59 is completely conserved evolutionarily among mammals.

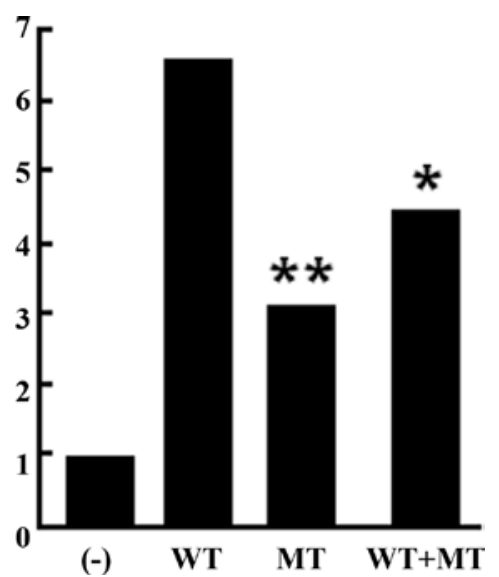

Figure 4. Transcriptional activity of the mutant NKX2-5 on the ANP promoter. COS-7 cells were transfected with $0.2 \mu \mathrm{g}$ of wild-type or mutant pEFSANKX2-5 expression vector, $0.2 \mu \mathrm{g}$ of ANP(-2600)-Luc reporter construct, and $0.04 \mu \mathrm{g}$ of pGL4.75 control reporter vector. For co-transfection experiments, $0.1 \mu \mathrm{g}$ of wild-type pEFSA-NKX2-5, $0.1 \mu \mathrm{g}$ of mutant pEFSA-NKX2-5, $0.2 \mu \mathrm{g}$ of ANP(-2600)-Luc, and $0.04 \mu \mathrm{g}$ of pGL4.75 were used. The activity of the ANP promoter was presented as fold activation of the firefly luciferase relative to the Renilla luciferase. Values are the mean \pm SD of data from three independent experiments performed in triplicate. ${ }^{*} \mathrm{P}<0.001$ and ${ }^{* *} \mathrm{P}<0.0001$, respectively, when compared with the wild-type NKX2-5.

\section{Discussion}

In the present study, we report a previously unrecognized NKX2-5 missense mutation identified in a family with congenital VSD. The novel heterozygous mutation was present in all 
the affected family members alive but absent in the unaffected relatives examined and in the 400 normal chromosomes from a matched control population. A cross-species alignment of the NKX2-5 protein sequences demonstrated that the altered amino acid was evolutionarily highly conserved among mammals. Functional analysis displayed that the p.P59A mutation of NKX2-5 was associated with decreased transcriptional activity. Therefore, it is very likely that mutated NKX2-5 is responsible for the VSD in this family.

Our results are supported by the discoveries of other NKX2-5 mutations predisposing to congenital VSD. To date, at least 16 mutations in NKX2-5 (p.E21Q, p.Q22P, p.Q25C, p.E109X, p.T178M, p.Q149X, p.R142C, p.W185L, p.Y191C, p.L202fs, p.R216C, p.A219V, p.Y259X, p.A323T, p.L171P, and p.R190H) have been detected in patients with isolated or syndromic VSD, showing that although NKX2-5 mutations are involved in a long list of cardiac malformations, one of frequent phenotypes ascribed to a mutation in NKX2-5 is VSD $(11,19-24)$. In most of these patients, the VSD-related mutations are familial, whereas sporadic cases remain relatively rare $(11,19-24)$. Similar to these findings, the mutation prevalence of over $8 \%(1 / 12)$ in our patients with positive family history suggests that the NKX2-5 mutations could be a major cause of familial VSD. Notably, remarkable genetic heterogeneity of VSD was substantiated by an inability to observe mutations in roughly $99 \%$ of our cohort patients, despite somatic NKX2-5 mutations as a potential mechanism of VSD in some patients (25-27). Therefore, the contribution of genes other than NKX2-5 to VSD pathogenesis seems likely.

Mutations in other transcription factors associated with cardiogenesis, such as GATA4, TBX5, and GATA6 (28-33), as well as mutations in cardiac structural proteins such as troponin I type 3 (TNNI3) and $\alpha$ myosin heavy chain (MYH6) have been identified in VSD patients $(34,35)$. However, so far, only $N K X 2-5$ mutations have been reported to cause a cardiac septal defect phenotype and the development of an atrioventricular block (18). The most common two phenotypes caused by mutated $N K X 2-5$ are cardiac septal defect and atrioventricular conduction disturbance (17), suggesting the pivotal role of $N K X 2-5$ not only in the morphogenesis of the heart, but also in the development of the cardiac conduction system. In the present study, a compound phenotype of VSD and an atrioventricular block were observed in 2/5 family members who carried the identified mutation of $N K X 2-5$. The atrioventricular block appeared to be progressive with increasing age in each individual as previously described $(11,14,23,36,37)$. As atrioventricular block is a possible cause of sudden cardiac death, a molecular genetic scanning will appear to be most favorable in identifying individuals at risk for life-threatening dysrhythmias. When an individual carrying an NKX2-5 mutation is identified, it is imperative for health care professionals to carefully monitor such a case that may currently present no symptoms, or to spontaneously close or surgically correct VSD, offering accurate and timely therapy.

Association of impaired NKX2-5 with increased susceptibility to VSD and atrioventricular block has been revealed in animal models. The homeobox-containing transcription factor encoded by the tinman gene, a homologue of NKX2-5, is expressed in the dorsal vessel (an insect equivalent of the vertebrate heart) of the fruit fly Drosophila melanogaster and targeted disruption of tinman leads to the lethal failure of the vessel formation (38). In Xenopus, expression of a similar DNA-non-binding mutant of $N K X 2-5$ has been shown to exert dominant negative effects on embryos, exhibiting small heart or no heart formation (39). In mice, $N K X 2-5$ is highly expressed in the early heart progenitor cells in both primary and secondary heart fields during embryogenesis and continues to be expressed at a high level in the heart through adulthood. A transiently elevated expression of $N K X 2-5$ is observed in specialized myocardial conduction cells during the development of the cardiac conduction system (17). In transgenic mice expressing a DNA binding-impaired mutant of the mouse NKX2-5 (I183P), under the $\beta$-myosin heavy chain promoter, the accumulation of mutant protein in the embryo, neonate, and adult myocardium resulted in progressive and profound cardiac conduction defects and heart failure (40). Targeted disruption of $N K X 2-5$ in mice results in embryonic lethality at about ED9.5, with aberrant heart morphogenesis and growth retardation $(41,42)$. Mice heterozygous for $N K X 2-5$-null alleles were predisposed to cardiac septal dysmorphogenesis and abnormal atrioventricular conduction (12). Ventricularrestricted NKX2-5 knockout around ED8.0 to ED8.5, created by crossing floxed-NKX2-5 mice with myosin light chain $2 \mathrm{v}$-Cre knock-in mice, postnatally resulted in progressive and advanced conduction defects and in left ventricular hypertrophy (14). In addition, perinatal loss of NKX2-5 leads to rapid conduction and contraction defects by regulating the expression of several ion channel genes (43). Together, these experimental results from animals imply that $N K X 2-5$ mutations are involved in a variety of congenital cardiac abnormalities including VSD and progressive conduction defects in humans.

Previous investigations have demonstrated that NKX2-5 is an upstream regulator or transcriptional activator of other genes expressed during cardiac development including atrial natriuretic factor gene, brain natriuretic peptide gene, and $\alpha$-cardiac actin gene $(42,44,45)$. NKX2-5 binding to target DNA may occur in conjunction with other partners including transcription factor GATA4 and serum response factor (45-47), and the synergistic transcriptional activation mediated by NKX2-5 and GATA4 has been ascertained (48). Therefore, the functional characteristics of the NKX2-5 mutations can be explored by analysis of the transcriptional activity of the ANP promoter in cells transfected with $N K X 2-5$ mutants in contrast to wild-type. Up to now, a total of 8 VSD-related NKX2-5 mutations (p.Q25C, p.T178M, p.Q149X, p.R142C, p.Y191C, p.Y259X, p.L171P, and p.R190H) have been characterized and all the mutations have loss-of function effects on the transcriptional activity of the ANP promoter except for p.Q25C, which has little effect $(22,49-51)$. Similarly, the novel p.P59A mutation of NKX2-5 identified in our familial VSD patients demonstrates a decreased transcriptional activity on the downstream gene. These findings indicate that haploinsufficiency or the dominant negative effect of NKX2-5 mutations are the major pathophysiological mechanisms underlying congenital VSD.

In conclusion, the current study links a novel mutation in the cardiac transcription factor NKX2-5 to familial VSD as well as to atrioventricular block. Furthermore, it provides new insight into the molecular mechanism implicated in the pathogenesis of VSD, implying potential implications for the 
genetic diagnosis and gene-specific therapy for this common disease in infancy.

\section{Acknowledgements}

We are indebted to participants for their dedication to the study. This study was supported in part by grants from the National Natural Science Fund of China (81070153, 30570768, and 30700776), the Natural Science Fund of Shanghai, China (10ZR1433100, 10ZR1428000, and 10JC1414002), and the National Basic Research Program of China (2010CB912604).

\section{References}

1. Lloyd-Jones D, Adams R, Carnethon M, et al: American Heart Association Statistics Committee and Stroke Statistics Subcommittee: Heart disease and stroke statistics-2009 update: a report from the American Heart Association Statistics Committee and Stroke Statistics Subcommittee. Circulation 119: e21-e181, 2009.

2. Minette MS and Sahn DJ: Ventricular septal defects. Circulation 114: 2190-2197, 2006.

3. Sommer RJ, Hijazi ZM and Rhodes JF Jr: Pathophysiology of congenital heart disease in the adult: part I: Shunt lesions. Circulation 117: 1090-1099, 2008.

4. McQuillen PS and Miller SP: Congenital heart disease and brain development. Ann NY Acad Sci 1184: 68-86, 2010.

5. Walsh EP: Interventional electrophysiology in patients with congenital heart disease. Circulation 115: 3224-3234, 2007.

6. Walsh EP and Cecchin F: Arrhythmias in adult patients with congenital heart disease. Circulation 115: 534-545, 2007.

7. Yap SC and Harris L: Sudden cardiac death in adults with congenital heart disease. Expert Rev Cardiovasc Ther 7: 1605-1620, 2009.

8. Jenkins KJ, Correa A, Feinstein JA, et al: American Heart Association Council on Cardiovascular Disease in the Young: Noninherited risk factors and congenital cardiovascular defects: current knowledge: a scientific statement from the American Heart Association Council on Cardiovascular Disease in the Young: endorsed by the American Academy of Pediatrics. Circulation 115: 2995-3014, 2007.

9. Pierpont ME, Basson CT, Benson DW Jr, et al: American Heart Association Congenital Cardiac Defects Committee, Council on Cardiovascular Disease in the Young: Genetic basis for congenital heart defects: current knowledge: a scientific statement from the American Heart Association Congenital Cardiac Defects Committee, Council on Cardiovascular Disease in the Young: endorsed by the American Academy of Pediatrics. Circulation 115: 3015-3038, 2007.

10. Pemberton VL, McCrindle BW, Barkin S, et al: Report of the National Heart, Lung, and Blood Institute's Working Group on obesity and other cardiovascular risk factors in congenital heart disease. Circulation 121: 1153-1159, 2010.

11. Schott JJ, Benson DW, Basson CT, et al: Congenital heart disease caused by mutations in the transcription factor NKX2-5. Science 281: 108-111, 1998.

12. Biben C, Weber R, Kesteven S, et al: Cardiac septal and valvular dysmorphogenesis in mice heterozygous for mutations in the homeobox gene Nkx2-5. Circ Res 87: 888-895, 2000.

13. Hiroi Y, Kudoh S, Monzen K, et al: Tbx5 associates with Nkx2-5 and synergistically promotes cardiomyocyte differentiation. Nat Genet 28: 276-280, 2001.

14. Pashmforoush M, Lu JT, Chen $\mathrm{H}$, et al: $\mathrm{Nkx} 2-5$ pathways and congenital heart disease; loss of ventricular myocyte lineage specification leads to progressive cardiomyopathy and complete heart block. Cell 117: 373-386, 2004.

15. Prall OW, Menon MK, Solloway MJ, et al: An Nkx2-5/Bmp2/ Smad1 negative feedback loop controls heart progenitor specification and proliferation. Cell 128: 947-959, 2007.

16. Moskowitz IP, Kim JB, Moore ML, et al: A molecular pathway including Id2, Tbx5, and Nkx2-5 required for cardiac conduction system development. Cell 129: 1365-1376, 2007.

17. Akazawa $\mathrm{H}$ and Komuro I: Cardiac transcription factor $\mathrm{Csx} /$ Nkx2-5: Its role in cardiac development and diseases. Pharmacol Ther 107: 252-268, 2005.
18. Stallmeyer B, Fenge H, Nowak-Göttl U and Schulze-Bahr E: Mutational spectrum in the cardiac transcription factor gene NKX2.5 (CSX) associated with congenital heart disease. Clin Genet 78: 533-540, 2010.

19. Benson DW, Silberbach GM, Kavanaugh-McHugh A, et al: Mutations in the cardiac transcription factor NKX2.5 affect diverse cardiac developmental pathways. J Clin Invest 104: 1567-1573, 1999.

20. Gutierrez-Roelens I, Sluysmans T, Gewillig M, Devriendt K and Vikkula M: Progressive AV-block and anomalous venous return among cardiac anomalies associated with two novel missense mutations in the CSX/NKX2-5 gene. Hum Mutat 20: 75-76, 2002.

21. McElhinney DB, Geiger E, Blinder J, Benson DW and Goldmuntz E: NKX2.5 mutations in patients with congenital heart disease. J Am Coll Cardiol 42: 1650-1655, 2003.

22. Kasahara $\mathrm{H}$ and Benson DW: Biochemical analyses of eight NKX2.5 homeodomain missense mutations causing atrioventricular block and cardiac anomalies. Cardiovasc Res 64: 40-51, 2004.

23. Sarkozy A, Conti E, Neri C, et al: Spectrum of atrial septal defects associated with mutations of NKX2.5 and GATA4 transcription factors. J Med Genet 42: e16, 2005.

24. Pabst S, Wollnik B, Rohmann E, et al: A novel stop mutation truncating critical regions of the cardiac transcription factor NKX2-5 in a large family with autosomal-dominant inherited congenital heart disease. Clin Res Cardiol 97: 39-42, 2008.

25. Reamon-Buettner SM, Hecker H, Spanel-Borowski K, Craatz S, Kuenzel E and Borlak J: Novel NKX2-5 mutations in diseased heart tissues of patients with cardiac malformations. Am J Pathol 164: 2117-2125, 2004.

26. Reamon-Buettner SM and Borlak J: Somatic NKX2-5 mutations as a novel mechanism of disease in complex congenital heart disease. J Med Genet 41: 684-690, 2004.

27. Draus JM Jr, Hauck MA, Goetsch M, Austin EH III, TomitaMitchell A and Mitchell ME: Investigation of somatic NKX2-5 mutations in congenital heart disease. J Med Genet 46: 115-122, 2009.

28. Garg V, Kathiriya IS, Barnes R, et al: GATA4 mutations cause human congenital heart defects and reveal an interaction with TBX5. Nature 424: 443-447, 2003.

29. Tomita-Mitchell A, Maslen CL, Morris CD, Garg V and Goldmuntz E: GATA4 sequence variants in patients with congenital heart disease. J Med Genet 44: 779-783, 2007.

30. Basson CT, Bachinsky DR, Lin RC, et al: Mutations in human TBX5 cause limb and cardiac malformation in Holt-Oram syndrome. Nat Genet 15: 30-35, 1997.

31. Li QY, Newbury-Ecob RA, Terrett JA, et al: Holt-Oram syndrome is caused by mutations in TBX5, a member of the Brachyury (T) gene family. Nat Genet 15: 21-29, 1997.

32. Maitra M, Koenig SN, Srivastava D and Garg V: Identification of GATA6 sequence variants in patients with congenital heart defects. Pediatr Res 68: 281-285, 2010.

33. Lin X, Huo Z, Liu X, et al: A novel GATA6 mutation in patients with tetralogy of Fallot or atrial septal defect. J Hum Genet 55: 662-667, 2010.

34. Yang SW, Hitz MP and Andelfinger G: Ventricular septal defect and restrictive cardiomyopathy in a paediatric TNNI3 mutation carrier. Cardiol Young 20: 574-576, 2010.

35. Granados-Riveron JT, Ghosh TK, Pope M, et al: Alpha-cardiac myosin heavy chain (MYH6) mutations affecting myofibril formation are associated with congenital heart defects. Hum Mol Genet 19: 4007-4016, 2010.

36. Hirayama-Yamada K, Kamisago M, Akimoto K, et al: Phenotypes with GATA4 or NKX2.5 mutations in familial atrial septal defect. Am J Med Genet A 135: 47-52, 2005.

37. Hosoda T, Komuro I, Shiojima I, et al: Familial atrial septal defect and atrioventricular conduction disturbance associated with a point mutation in the cardiac homeobox gene CSX/KX2-5 in a Japanese patient. Jpn Circ J 63: 425-426, 1999.

38. Bodmer R: The gene, tinman, is required for specification of the heart and visceral muscles in Drosophilia. Development 118: 719-729, 1993.

39. Grow MW and Krieg PA: Tinman function is essential for vertebrate heart development: elimination of cardiac differentiation by dominant inhibitory mutants of the tinman-related genes, XNkx2-3 and XNkx2-5. Dev Biol 204: 187-196, 1998.

40. Kasahara $\mathrm{H}$, Wakimoto $\mathrm{H}$, Liu M, et al: Progressive atrioventricular conduction defects and heart failure in mice expressing a mutant Csx/Nkx2.5 homeoprotein. J Clin Invest 108: 189-201, 2001. 
41. Lyons I, Parsons LM, Hartley L, Li R, Andrews JE, Robb L and Harvey RP: Myogenic and morphogenetic defects in the heart tubes of murine embryos lacking the homeo box gene Nkx2-5. Genes Dev 9: 1654-1666, 1995.

42. Tanaka M, Chen Z, Bartunkova S, Yamasaki N and Izumo S: The cardiac homeobox gene Csx/Nkx2.5 lies genetically upstream of multiple genes essential for heart development. Development 126: 1269-1280, 1999.

43. Briggs LE, Takeda M, Cuadra AE, et al: Perinatal loss of Nkx2-5 results in rapid conduction and contraction defects. Circ Res 103: 580-590, 2008.

44. Durocher D, Chen CY, Ardati A, Schwartz RJ and Nemer M: The atrial natriuretic factor promoter is a downstream target for Nkx2-5 in the myocardium. Mol Cell Biol 16: 4648-4655, 1996.

45. Chen CY, Croissant J, Majesky M, Topouzis S, McQuinn T, Frankovsky MJ and Schwartz RJ: Activation of the cardiac alpha-actin promoter depends upon serum response factor Tinman homologue, Nkx2-5, and intact serum response elements. Dev Genet 19: 119-130, 1996.

46. Durocher D, Charron F, Warren R, Schwartz RJ and Nemer M: The cardiac transcription factors $N k \times 2-5$ and GATA-4 are mutual cofactors. EMBO J 16: 5687-5696, 1997.
47. Lee Y, Shioi T, Kasahara H, Jobe SM, Wiese RJ, Markham BE and Izumo S: The cardiac tissue-restricted homeobox protein Cs $x / N k x 2-5$ physically associates with the zinc finger protein GATA4 and cooperatively activates atrial natriuretic factor gene expression. Mol Cell Biol 18: 3120-3129, 1998.

48. Shiojima I, Komuro I, Oka T, et al: Context-dependent transcriptional cooperation mediated by cardiac transcription factors Csx/Nkx2.5 and GATA-4. J Biol Chem 274: 8231-8239, 1999.

49. Zhu W, Shiojima I, Hiroi Y, et al: Functional analyses of three $\mathrm{Csx} / \mathrm{Nkx}-2.5$ mutations that cause human congenital heart disease. J Biol Chem 275: 35291-35296, 2000.

50. Kasahara H, Lee B, Schott JJ, Benson DW, Seidman JG, Seidman CE and Izumo S: Loss of function and inhibitory effects of human CSX/NKX2.5 homeoprotein mutations associated with congenital heart disease. J Clin Invest 106: 299-308, 2000.

51. Inga A, Reamon-Buettner SM, Borlak J and Resnick MA: Functional dissection of sequence-specific NKX2-5 DNA binding domain mutations associated with human heart septation defects using a yeast-based system. Hum Mol Genet 14: 1965-1975, 2005 . 